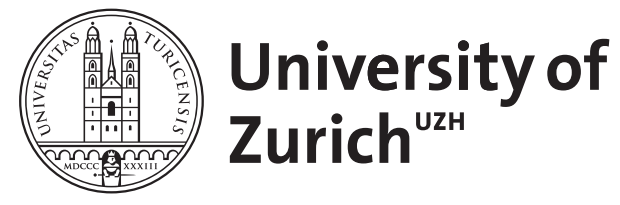

\title{
Möbius characterization of hemispheres
}

Foertsch, T; Schroeder, Viktor

\begin{abstract}
In this paper we generalize the Möbius characterization of metric spheres as obtained in
\end{abstract} Foertsch and Schroeder [4] to a corresponding Möbius characterization of metric hemispheres.

DOI: https://doi.org/10.1007/s00013-012-0399-6

Posted at the Zurich Open Repository and Archive, University of Zurich ZORA URL: https://doi.org/10.5167/uzh-71202

Journal Article

Published Version

Originally published at:

Foertsch, T; Schroeder, Viktor (2012). Möbius characterization of hemispheres. Archiv der Mathematik, 99(1):81-89.

DOI: https://doi.org/10.1007/s00013-012-0399-6 


\title{
Möbius characterization of hemispheres
}

\author{
Thomas Foertsch And VikTor Schroeder
}

\begin{abstract}
In this paper we generalize the Möbius characterization of metric spheres as obtained in Foertsch and Schroeder [4] to a corresponding Möbius characterization of metric hemispheres.
\end{abstract}

1. Introduction. This paper is a continuation of [5] where we studied and classified Ptolemy circles and Ptolemy segments.

Recall that a metric space $(X, d)$ is called a Ptolemy metric space if the inequality

$$
d\left(x_{1}, x_{3}\right) d\left(x_{2}, x_{4}\right) \leq d\left(x_{1}, x_{2}\right) d\left(x_{3}, x_{4}\right)+d\left(x_{1}, x_{4}\right) d\left(x_{3}, x_{2}\right) .
$$

holds for all quadruples $x_{1}, x_{2}, x_{3}, x_{4} \in X$.

We call a subset $\sigma \subset X$ a Ptolemy circle, if $\sigma$ is homeomorphic to $S^{1}$ and for any four points $x_{1}, x_{2}, x_{3}, x_{4}$ on $\sigma$ (in this order) we have equality in Eq. (1). Similarly we call a subset $I \subset X$ a Ptolemy segment, if $I$ is homeomorphic to a closed interval and for any four points $x_{1}, x_{2}, x_{3}, x_{4}$ on $\sigma$ (in this order) we have equality in Eq. (1).

The standard examples of Ptolemy metric spaces are the Euclidean space $\mathbb{E}^{n}$ or the sphere $S^{n} \subset \mathbb{E}^{n+1}$ with the induced (chordal) metric, which we denote by $d_{0}$. As shown in [2], further examples of Ptolemy metric spaces are the boundaries at infinity of $\mathrm{CAT}(-1)$-spaces when endowed with their Bourdon metrics. Also compare [1] for more examples of Ptolemy spaces.

In this paper we give a Möbius characterization of Ptolemy spaces with the property that every triple of points is contained in a Ptolemy segment. This generalizes the main result from [4], which says

Theorem 1.1. Let $(X, d)$ be a compact extended Ptolemy metric space which contains at least three points. If any three points in $X$ lie on a Ptolemy circle, then $X$ is Möbius equivalent to $\left(S^{n}, d_{0}\right)$ for some $n \in \mathbb{N}$. 
Here we prove a generalized version. We use the terminology of [5]. In particular we use the notion of extended metric spaces, which are metric spaces which may have one additional point at infinity.

Theorem 1.2. Let $(X, d)$ be a compact extended Ptolemy metric space containing at least three points. If any three points in $X$ lie on a circle or on a Ptolemy segment, then $(X, d)$ is Möbius equivalent to either some $\left(S^{n}, d_{0}\right)$ or to some hemisphere $\left(S_{+}^{n}, d_{0}\right)$ for some $n \in \mathbb{N}$.

It is a pleasure to thank Swiatoslaw Gal for a hint concerning Lemma 2.6. We also want to thank the referee for useful remarks and corrections.

2. Preliminaries and auxiliary results. For basic definitions compare [5].

2.1. Metric involution. Let $(X, d)$ be an extended Ptolemy space and $z \in X$ which is not infinitely remote. Now define $d_{z}: X \times X \rightarrow[0, \infty]$ by

$$
\begin{aligned}
& d_{z}(x, y)=\frac{d(x, y)}{d(z, x) d(z, y)} \quad \text { for } x, y \in X \backslash(\Omega(d) \cup\{z\}), \\
& d_{z}(x, \omega)=\frac{1}{d(z, x)} \quad \text { for } x \in X \backslash \Omega(d), \\
& d_{z}(z, x)=\infty \quad \text { for } x \in X \backslash\{z\}
\end{aligned}
$$

Since for $x, y, w \in X \backslash\{z\}$

$$
\begin{aligned}
& \left(d_{z}(x, y): d_{z}(y, w): d_{z}(x, w)\right)= \\
& \quad(d(x, y) d(z, w): d(x, z) d(y, w): d(x, w) d(y, z)) \in \Delta
\end{aligned}
$$

we see that $d_{z}$ satisfies the triangle inequality. It is easy to check that the identity $(X, d) \longrightarrow\left(X, d_{z}\right)$ is Möbius.

2.2. Convexity and Busemann functions. Recall that a geodesic metric space $(X, d)$ is called distance convex, if all its distance functions to points $z \in X$

$$
d(z, \cdot): X \longrightarrow \mathbb{R}_{0}^{+}, \quad x \mapsto d(z, x) \forall x \in X
$$

are convex, i.e., that their restriction to any geodesic segment in $(X, d)$ is convex.

A geodesic Ptolemy metric space is distance convex, which follows immediately from the Ptolemy inequality applied to points $z, x, m$, and $y$, where $m$ is a midpoint of $x$ and $y$, i.e. $d(x, m)=\frac{1}{2} d(x, y)=d(m, y)$.

Let $c:[0, \infty) \rightarrow X$ be a geodesic ray parameterized by arclength. As usual we define the Busemann function $b_{c}(x)=\lim _{t \rightarrow \infty}(d(x, c(t))-t)$.

If $X$ is a geodesic Ptolemy space, then $b_{c}$ is convex, being the limit of the convex functions $d(c(t), \cdot)-t$.

For more information on Busemann functions in geodesic Ptolemy spaces we refer the reader to [3].

2.3. Affine functions and the Hitzelberger-Lytchak theorem. Let $X$ be a geodesic metric space. For $x, y \in X$ we denote by $m(x, y)=\{z \in X \mid d(x, z)=$ $\left.d(z, y)=\frac{1}{2} d(x, y)\right\}$ the set of midpoints of $x$ and $y$. A map $f: X \rightarrow Y$ 
between two geodesic metric spaces is called affine, if for all $x, y \in X$, we have $f(m(x, y)) \subset m(f(x), f(y))$. Thus a map is affine if and only if it maps geodesics parameterized proportionally to arclength into geodesics parameterized proportionally to arclength. An affine map $f: X \rightarrow \mathbb{R}$ is called an affine function.

Definition 2.1. Let $X$ be a geodesic metric space. We say that affine functions on $X$ separate points, if for every $x, y \in X, x \neq y$, there exists an affine function $f: X \longrightarrow \mathbb{R}$ with $f(x) \neq f(y)$.

A variant of the following beautiful rigidity theorem, which is due to Hitzelberger and Lytchak, is a main tool in our argument.

Theorem 2.2. [6] Let $X$ be a geodesic metric space. If the affine functions on $X$ separate points, then $X$ is isometric to a convex subset of a (strictly convex) normed vector space.

For a proof of a variant of this statement, cf. Sect. 3.

2.4. Normed vector spaces. The other main ingredient when characterizing Ptolemy metric spaces with sufficiently many Ptolemy-circles or -segments is the following theorem due to Schoenberg.

Theorem 2.3. [7] A normed vector space $(V,\|\cdot\|)$ is a Ptolemy metric space if and only if it is Euclidean.

This, together with the fact that the Ptolemy condition is invariant under scaling, yields the

Corollary 2.4. An open subset of a normed vector space $(V,\|\cdot\|)$ is a Ptolemy space, if and only if $(V,\|\cdot\|)$ is Euclidean.

2.5. Rigidity of Ptolemy-circles and Ptolemy segments. In this section we point out that Ptolemy spaces, where any quadruple of points lies on a circle or a segment already is a circle or segment itself.

Proposition 2.5. An extended Ptolemy metric space with the property that through each four points of the space there exists a Ptolemy circle (Ptolemy segment) containing these points, is Möbius equivalent to $S^{1}$ (or the hemisphere $\left.S_{+}^{1}\right)$ when endowed with its chordal metric.

We assume first, that $X$ is a space such that for any allowed quadruple $\left(x_{1}, x_{2}, x_{3}, x_{4}\right)$ we have $\operatorname{crt}\left(x_{1}, x_{2}, x_{3}, x_{4}\right) \in \partial \Delta$, i.e. for all quadruples of points the Ptolemy equality holds. We choose some point $p \in X$ and consider the metric space $\left(X \backslash\{p\}, d_{p}\right)$. The Ptolemy equality implies now that for a triple of points in $X \backslash\{p\}$ we have equality in the triangle inequality.

Now we use the following elementary result, which we leave as an exercise.

Lemma 2.6. Let $Y$ be a metric space, such that for any triple of points we have equality in the triangle inequality. Then either $Y$ is a four point space $Y=\{x, y, z, w\}$ such that $d(x, y)=d(z, w)=a, d(x, z)=d(y, w)=b$ and $d(x, w)=d(y, z)=a+b$ for some $a, b>0$ or $Y$ is isometric to a subset of the real line $\mathbb{R}$. 
Since $X$ contains segments and hence has infinitely many points, it follows that $X$ is Möbius equivalent to $Y \cup\{\infty\}$, where $Y \subset \mathbb{R}$. Since Ptolemy segment spaces are connected, we immediately obtain Proposition 2.5.

3. Proof of the main result. In this section we prove Theorem 1.2.

The proof of this result is surprisingly much more involved than the proof of Theorem 1.1. The idea of the proof is the same, but there arise quite a number of technical problems.

The main step is to reduce the problem to the case, that $X$ is already a subset of the classical space $\left(\mathbb{S}^{n}, d_{0}\right)$. For the classical case our result can also be reformulated in the following way:

Proposition 3.1. Let $X \subset \mathbb{R}^{k} \cup\{\infty\}$ with $\infty \in X$ such that $X \backslash\{\infty\}$ is a closed subset of $\mathbb{R}^{k}$. Assume that through any three distinct points of $X$ there exists a circle segment through these points. Then $X \backslash\{\infty\}$ is contained in an affine subspace $H \subset \mathbb{R}^{k}$ (which could be $\mathbb{R}^{k}$ again), and $H \cap X$ is isometric either to $H$, to some closed half space in $H$ or to the complement of some open distance ball in $H$.

We will give a sketch of the proof at the end of the section.

We now come to the proof of Theorem 1.2. For simplicity we call a metric space a 3-point Ptolemy-segment space, if it is Ptolemy and every triple of points is contained in a segment.

Let $(X, d)$ be a compact 3-point Ptolemy segment space, and consider again a chosen point $z \in X$ and the locally compact metric space $M:=\left(X \backslash\{z\}, d_{z}\right)$.

Let $x, y \in M$ be distinct points. By assumption there exists a Ptolemy segment $\sigma$ in $X$ containing $x, y, z$. Using the arguments of Sect. 2.5 and Lemma 2.6 we see that $\sigma \backslash\{z\}$ is in the metric space $M$ isometric to a subset of the real line $\mathbb{R}$. Thus using some parameterization by arclength there exists an isometric map $c_{x y}: \mathbb{R} \backslash I \rightarrow M$, with $c_{x y}(0)=x, c_{x y}(d(x, y))=y$, where $I \subset \mathbb{R}$ is either empty or an open interval. We call the image $c_{x y}(\mathbb{R} \backslash I) \subset M$ a geodesic minus interval through $x$ and $y$. We do not know about uniqueness for the moment. The geodesic minus interval contains at least one ray. It may be that $I \subset[0, d(x, y)]$ and in this case, there may not be a geodesic between $x$ and $y$. Thus $M$ is in general not a geodesic space. In particular we cannot directly use the methods of [4]. Even if a geodesic exists between two points, we do not know uniqueness. We can also not use the Hitzelberger-Lytchak theorem. Instead we have to reprove the relevant results in our special situation.

We first have to state some properties of affine functions. Let $M$ be a (not necessarily geodesic) metric space. In our context $M$ is the space $\left(X \backslash\{z\}, d_{z}\right)$. For $x, y \in M$ we denote by $m(x, y)=\left\{z \in M \mid d(x, z)=d(z, y)=\frac{1}{2} d(x, y)\right\}$ the set of midpoints of $x$ and $y$. A function $f: X \rightarrow \mathbb{R}$ is called affine, if for all $x, y \in X$ and all $m \in m(x, y)$, we have $f(m)=\frac{1}{2}(f(x)+f(y))$.

Essential for our argument is that Busemann functions of geodesic rays are affine in this sense.

Proposition 3.2. A Busemann function $b: M \rightarrow \mathbb{R}$ is affine in this sense.

We postpone the proof and focus on the consequences. 
We set

$$
\mathcal{A}^{\prime}(M):=\{f: M \rightarrow \mathbb{R} \mid f \text { affine and Lipschitz }\} .
$$

$\mathcal{A}^{\prime}(M)$ is a Banach space, where $\|f\|$ is the optimal Lipschitz constant. We set $\mathcal{A}(M):=\mathcal{A}^{\prime}(M) / \sim$, where $f \sim g$ if $(f-g)$ is constant. By $[f]$ we denote the equivalence class of $f$. Then also $\mathcal{A}(M)$ is a Banach space, where $\|[f]\|=\|f\|$. Let $\mathcal{A}^{*}(M)$ be the Banach dual space of $\mathcal{A}(M)$ with the norm

$$
\|\rho\|=\sup _{[f] \in \mathcal{A}(M)} \frac{|\rho([f])|}{\|[f]\|} .
$$

For $x, y \in M$ let $E(x, y) \in \mathcal{A}^{*}(M)$ be the evaluation map $E(x, y)([f])=$ $f(x)-f(y)$. It follows directly from the definitions that $\|E(x, y)\| \leq d(x, y)$. For a given basepoint $o \in M$ we consider the map

$$
A_{o}: M \rightarrow \mathcal{A}^{*}(M), \quad x \mapsto E(x, o) .
$$

Note that $A_{o}$ is 1-Lipschitz since

$$
\left\|A_{o}(x)-A_{o}(y)\right\|=\|E(x, y)\| \leq d(x, y) .
$$

If $x, y \in M$ and $z \in m(x, y)$ then for all $[f] \in \mathcal{A}$ we have

$$
\begin{aligned}
E(z, o)[f] & =f(z)-f(o)=\frac{1}{2}(f(x)+f(y))-f(o) \\
& =\left(\frac{1}{2} E(x, o)+\frac{1}{2} E(y, o)\right)[f] .
\end{aligned}
$$

This implies

$$
A_{o}(m)=\frac{1}{2}\left(A_{o}(x)+A_{o}(y)\right) .
$$

We now show that $A_{o}$ is actually an isometric map. Let $x, y \in M$, then we have a geodesic minus interval $c_{x y}: \mathbb{R} \backslash I \rightarrow M$. Recall that $c_{x y}(\mathbb{R} \backslash I)$ contains at least one ray and let $b$ be the Busemann function of such a ray. Then $b$ is 1-Lipschitz and $|b(x)-b(y)|=d(x, y)$.

Hence

$$
\left\|A_{o}(x)-A_{o}(y)\right\|=\|E(x, y)\| \geq|E(x, y)([b])|=|b(y)-b(x)|=d(x, y) .
$$

Thus $A_{o}$ is an isometric embedding of $M$ into the Banach space $\mathcal{A}^{*}=\mathcal{A}^{*}(M)$. The Eq. (2) shows that the geodesic minus interval is mapped to an affine line minus interval in the Banach space $\mathcal{A}^{*}$.

For simplicity, we consider in the sequel $M$ as a subset of the vector space $\mathcal{A}^{*}$.

Lemma 3.3. Let $x_{0}, \ldots, x_{n} \in M \subset \mathcal{A}^{*}$ be finitely many points. Let $E$ be the affine hull of these points, i.e. the smallest affine subspace containing these points. Then $E \cap M$ contains an open set in the induced topology of $E$.

Proof. This is proven by induction on the dimension of $E$, where the case of dimension 0 is trivial. Assume now that the affine hull of $S=\left\{x_{0}, \ldots, x_{n}\right\}$ has dimension $(m+1)$. Then there exists a subset $S^{\prime} \subset S$, such that the dimension of the affine hull $E^{\prime}$ of $S^{\prime}$ is $m$ and by induction hypothesis there exists an open subset $U^{\prime} \subset E^{\prime}$, with $U^{\prime} \subset M$. Furthermore there exists a point $x \in S$ such that $x \in E \backslash E^{\prime}$. For every $u \in U^{\prime}$ consider the line minus interval 
$c_{x u}: \mathbb{R} \backslash I_{u} \rightarrow M \cap E$. We reparameterize these lines minus interval proportionally to arclength such that $c_{x u}(0)=x$ and $c_{x u}(1)=u$. Then $I_{u} \subset R \backslash\{0,1\}$ is an open interval. If for all $u \in U^{\prime}, I_{u} \cap[0,1]=\emptyset$, then $\bigcup_{u} c_{x u}((0,1)) \subset M$ is an open subset of $E$. If for some $u_{0}$ we have $I_{u_{0}} \cap[0,1] \neq \emptyset$ then $I_{u_{0}} \subset(0,1)$ and then also $I_{u} \subset(0,1)$ for all $u \in U^{\prime \prime}$, where $U^{\prime \prime}$ is an open neighborhood of $u_{0}$ in $U^{\prime}$. Then $\bigcup_{u \in U^{\prime \prime}} c_{x, u}((1, \infty)) \subset M$ is an open subset of $E$.

Lemma 3.4. For all finite subsets $S \subset M$, the affine hull of $S$ in $\mathcal{A}^{*}$ is a Euclidean subspace.

Proof. $E \cap M$ contains an open subset of $E$ by Lemma 3.3, and $E \cup M$ is a Ptolemy space. Thus, by Corollary 2.4 of Schoenberg's result, $E$ is a Euclidean space.

Lemma 3.5. Let $E$ be an affine Euclidean subspace of $\mathcal{A}^{*}$, then $(E \cap M) \cup\{\infty\}$ is a 3-point Ptolemy segment space.

Proof. Let $x_{1}, x_{2}, x_{3} \in(E \cap M) \cup\{\infty\}$ be distinct points. By assumption on $M$ there exists a Ptolemy segment $\sigma$ in $M \cup\{\infty\}$ containing these points. We have to show that $\sigma \subset E \cup\{\infty\}$. We actually show that $\sigma \subset E^{\prime} \cup\{\infty\}$, where $E^{\prime}$ is the affine hull of $\left\{x_{1}, x_{2}, x_{3}\right\}$. Let $x_{4} \in \sigma$, we show that $x_{4} \in E^{\prime} \cup\{\infty\}$. We can assume $x_{4} \neq \infty$ and furthermore that all four points are distinct. Take the affine hull $E^{\prime \prime}$ of $\left\{x_{1}, x_{2}, x_{3}, x_{4}\right\}$, then $E^{\prime} \subset E^{\prime \prime}$. By Lemma $3.4 E^{\prime \prime}$ is a Euclidean space and the four points satisfy the Ptolemy equality. By the classical Ptolemy theorem this implies that $x_{4}$ is contained in the affine hull of the three other points.

Lemma 3.6. The affine hull of $M$ is contained in a finite dimensional Euclidean space.

Proof. It suffices to show that there is a number $k \in \mathbb{N}$, such that the dimension of the affine hull of any finite subset $S \subset M$ is bounded by $k$. If we assume the contrary, there are points $x_{0}, x_{1}, \ldots \in M$, such that the affine hull of $x_{0}, \ldots, x_{n}$ is an $n$-dimensional Euclidean affine subspace $E^{n} \subset \mathcal{A}^{*}$. By Lemma 3.5, $M \cap E^{n}$ is a 3-point Ptolemy segment space, and hence $M \cap E^{n}$ is either $E^{n}$, isometric to a closed half space or isometric to the complement of a distance ball in $E^{n}$ by Proposition 3.1. In every case $E \cap M$ contains a complete hyperplane $P^{n-1}$ of dimension $(n-1)$ through the point $x_{0}$. We can arrange the hyperplanes such that $P^{n} \subset P^{n+1}$ for all $n$. Since $M$ is locally compact, this is a contradiction.

Now we can finish the proof of Theorem 1.2. By Lemma 3.6, $M$ can be considered as a subset of the classical space $\mathbb{R}^{n} \cup\{\infty\}$ and for this case the result follows from Proposition 3.1.

Now we give the

Proof. (of Proposition 3.2) Let $c:[0, \infty) \longrightarrow X \backslash\{z\}$ be an arbitrary geodesic ray in $\left(X \backslash\{z\}, d_{z}\right)$ and let $b$ be a Busemann function of that ray. Consider 
$x, y, m \in X$ with $a:=d(x, y) / 2=d(x, m)=d(m, y)$. Since Busemann functions are convex (cf. Sect. 2.2), we have $b(m) \leq \frac{1}{2}(b(x)+b(y))$. Therefore, it remains to prove that

$$
b(m) \geq \frac{1}{2}(b(x)+b(y)) .
$$

Consider $w_{i}=c(i)$ for $i \rightarrow \infty$. By assumption there exists a Ptolemy segment $\sigma_{i}$ containing $x, y, w_{i}$.

We restrict the segment such that it contains two of these points as boundary points and one as an interior point. After a choice of a subsequence, we can assume that one of the following three cases occurs: all $\sigma_{i}$ have

(i) $y$ as interior point, (i') $x$ as interior point, (ii) $w_{i}$ as interior point.

The cases (i) and (i') are symmetric, so we only consider (i) and (ii).

$C A S E(i)$ :

We consider the subsegment of this circle which contains $x$ and $w_{i}$ as boundary points and $y$ as interior point. On this segment we consider the orientation that $x<y<w_{i}$ and we choose for $i$ large enough $u_{i} \in \sigma_{i}$ with $x<y<u_{i}<w_{i}$, such that $d\left(y, u_{i}\right)=a$, and hence $d\left(x, u_{i}\right) \leq 3 a$. By the Ptolemy equality on the segment we have

$$
3 a d\left(y, w_{i}\right) \geq d\left(y, w_{i}\right) d\left(x, u_{i}\right)=2 a d\left(u_{i}, w_{i}\right)+a d\left(x, w_{i}\right)
$$

and hence

$$
d\left(y, w_{i}\right) \geq \frac{2}{3} d\left(u_{i}, w_{i}\right)+\frac{1}{3} d\left(x, w_{i}\right) .
$$

Since $(X \backslash\{z\})$ is locally compact, some subsequence converges, thus we can assume $u_{i} \rightarrow u$ and $x, y, u$ lie on a geodesic.

Since $u_{i} \rightarrow u$, the Inequality (4) implies in the limit for the Busemann function

$$
b(y) \geq \frac{2}{3} b(u)+\frac{1}{3} b(x) .
$$

Since $x, y, u$ are on a geodesic and $d(y, u)=a$, we have $d(x, u)=3 a$. By triangle inequalities this implies $d(m, u)=2 a$, and hence $y$ is a midpoint of $m$ and $u$. This again implies

$$
b(y) \leq \frac{1}{2}(b(m)+b(u)) .
$$

Now an easy computation shows that Inequalities (5) and (6) imply the desired estimate (3).

CASE (ii):

There exists a Ptolemy segment $\sigma_{i}$ with boundary points $x$ and $y$ and interior point $w_{i}$. Here we choose the orientation, such that $y<w_{i}<x$ and again we choose $u_{i} \in \sigma_{i}$, with $y<u_{i}<w_{i}<x$ such that $\left|y u_{i}\right|=a$. The Ptolemy equality for this segment implies again

$$
3 a d\left(y, w_{i}\right) \geq d\left(y, w_{i}\right) d\left(x, u_{i}\right)=2 a d\left(u_{i}, w_{i}\right)+\operatorname{ad}\left(x, w_{i}\right)
$$

and hence we again obtain estimate (4).

Again for a subsequence $u_{i} \rightarrow u$ we obtain (5). 
The Ptolemy equality for $\sigma_{i}$ says

$$
d\left(x, u_{i}\right) d\left(w_{i}, y\right)=d(x, y) d\left(w_{i}, u_{i}\right)+d\left(y, u_{i}\right) d\left(w_{i}, x\right),
$$

this implies again for $i \rightarrow \infty$, that $d(x, u)=d(x, y)+d(y, u)=3 a$, which again implies that $y$ is a midpoint of $m$ and $u$ and hence we have Eq. (6) again.

In the same way as above we obtain Eq. (3).

Finally we give the

Proof. (of Proposition 3.1) Consider $X \subset \mathbb{R}^{k} \cup\{\infty\}$ as in the assumption. Let $H$ be the smallest affine subspace of $\mathbb{R}^{k}$ containing $X \backslash\{\infty\}$. To simplify the notation we assume that $H=\mathbb{R}^{k}$ (otherwise the result follows by induction).

Case 1. $X \backslash\{\infty\}=\mathbb{R}^{k}$, then we are finished.

Case 2. Assume that $X \backslash\{\infty\}$ is contained in some halfspace. By changing coordinates we may assume $X \backslash\{\infty\} \subset \mathbb{R}^{k-1} \times[0, \infty)$. Let $x, y \in X \backslash\{\infty\}$ and assume that for the last coordinates we have $x_{k}<y_{k}$. Since $x, y, \infty$ are on a circle-segment (i.e. line-segment) contained in $X$, but $X \backslash\{\infty\}$ does not intersect the lower half space, we see that the semiline $x+t(y-x), t \geq 0$ is completely contained in $X$.

It is elementary (but somewhat cumbersome) to prove the following fact:

Let $B \subset \mathbb{R}^{k-1} \times[0, \infty)$ be a closed subset, with the following properties:

(1) $B$ contains $(k+1)$ affinely independent points.

(2) if $x, y \in B$ with $x_{k}<y_{k}$, then also $x+t(y-x) \in B$ for $t \geq 0$.

Then $B=\mathbb{R}^{k-1} \times[a, \infty)$ for some $a \geq 0$.

This proves the second case.

Case 3. In the remaining case we consider that $X \backslash\{\infty\}$ is not contained in a half space and not the complete $\mathbb{R}^{k}$. Then there exists an open distance ball $D \subset \mathbb{R}^{k} \backslash X$, such that there exists a point $x \in \partial D \cap X$. By changing coordinates we can assume that $x=0$ is the origin. Now apply the involution $x \mapsto \frac{x}{\|x\|}, 0 \mapsto \infty, \infty \mapsto 0$, which is a Möbius map and maps circles to circles. Under this involution $D$ goes to an open half space and hence we have reduced this case to Case 2.

\section{References}

[1] Th. Foertsch, A. Lytchak, and V. Schroeder, Nonpositive curvature and the Ptolemy inequality, Int. Math. Res. Not. IMRN 2007, 15 pp.

[2] Th. Foertsch and V. Schroeder, Hyperbolicity, CAT(-1)-spaces and the Ptolemy Inequality, Math. Ann. 350 (2011), 339-356.

[3] Th. Foertsch And V. Schroeder, Group actions on geodesic Ptolemy spaces, Trans. Amer. Math. Soc. 363 (2011), 2891-2906.

[4] Th. Foertsch And V. Schroeder, Metric Möbius Geometry and the Characterization of Spheres, to appear in Manuscripta Math. 
[5] Th. Foertsch and V. Schroeder, Ptolemy Circles and Ptolemy Segments, Archiv der Mathematik, to appear.

[6] P. Hitzelberger And A. Lytchak, Spaces with many affine functions, Proc. AMS 135 (2007), 2263-2271.

[7] I.J. Schoenberg, A remark on M. M. Day's characterization of inner-product spaces and a conjecture of L. M. Blumenthal, Proc. Amer. Math. Soc. 3 (1952), 961-964.

T. Foertsch And V. Schroeder

Institut für Mathematik, Universität Zürich,

Winterthurer Strasse 190, 8057 Zurich,

Switzerland

e-mail: foertschthomas@googlemail.com

V. Schroeder

e-mail: vschroed@math.uzh.ch

Received: 19 April 2012 